\title{
PROCESSING, CATALOGUING AND DISTRIBUTION OF UAS IMAGES IN NEAR REAL TIME
}

\author{
I. Runkel
}

GEOSYSTEMS GmbH - i.runkel@geosystems.de

KEY WORDS: Near real time, raster data management system, OGC services, fully automated workflow for data pre-processing and data dissemination

\begin{abstract}
:
Why are UAS such a hype? UAS make the data capture flexible, fast and easy. For many applications this is more important than a perfect photogrammetric aerial image block.

To ensure, that the advantage of a fast data capturing will be valid up to the end of the processing chain, all intermediate steps like data processing and data dissemination to the customer need to be flexible and fast as well. GEOSYSTEMS has established the whole processing workflow as server/client solution. This is the focus of the presentation.

Depending on the image acquisition system the image data can be down linked during the flight to the data processing computer or it is stored on a mobile device and hooked up to the data processing computer after the flight campaign. The image project manager reads the data from the device and georeferences the images according to the position data. The meta data is converted into an ISO conform format and subsequently all georeferenced images are catalogued in the raster data management System ERDAS APOLLO. APOLLO provides the data, respectively the images as an OGC-conform services to the customer.

Within seconds the UAV-images are ready to use for GIS application, image processing or direct interpretation via web applications - where ever you want.

The whole processing chain is built in a generic manner. It can be adapted to a magnitude of applications. The UAV imageries can be processed and catalogued as single ortho imges or as image mosaic. Furthermore, image data of various cameras can be fusioned. By using WPS (web processing services) image enhancement, image analysis workflows like change detection layers can be calculated and provided to the image analysts. The processing of the WPS runs direct on the raster data management server. The image analyst has no data and no software on his local computer.

This workflow is proven to be fast, stable and accurate. It is designed to support time critical applications for security demands - the images can be checked and interpreted in near real-time. For sensible areas it gives you the possibility to inform remote decision makers or interpretation experts in order to provide them situations awareness, wherever they are. For monitoring and inspection tasks it speeds up the process of data capture and data interpretation.

The fully automated workflow of data pre-processing, data georeferencing, data cataloguing and data dissemination in near real time was developed based on the Intergraph products ERDAS IMAGINE, ERDAS APOLLO and GEOSYSTEMS METAmorph!IT. It is offered as adaptable solution by GEOSYSTEMS GmbH.
\end{abstract}

\section{INTRODUCTION}

The motivation for this project is driven by the fact, that the data capture of imageries, using UAS, has a strong focus in the scientific as well the commercial sector. But to complete the full process of a UAS, the subsequent data management of the recorded imageries, respectively the derived products like ortho imageries or DSMs needs to be investigated as well.

One focus of the SELSAS-Hym project - financed by the German Army - was to establish the full automated sequence from data capture to data management and data dissemination under the consideration of near real-time processing. The findings of the defence relevant aspects are adaptable to all kind of UAS workflows, if time is a critical factor.

This paper describes the main aspects of SELSAS-HyM project. The focus of this presentation is the image processing part of the project.

\section{VALUE ADDING DUE TO COMBINATION}

The complete UAS processing starts with the flight campaign with all relevant tasks, which are firmly related to the flight carrier, like flight planning, data capture, GPS recording, and so on.

Once the data are recorded, image processing is the next step. This is highly dependent from the final task of the mission. There are all kinds of processing steps - from simple stitching to photogrammetric processing of ortho images. Many business models for UAS processing are finished at that point. But to generate a sustainable business model it is recommended to proceed with subsequent steps, like catalogue the images in an OGC catalogue together with relevant metadata (geometric and keyword metadata) to enable an easy access to the data as OGC services, either in web clients or in GIS-packages. This is a future-oriented approach to UAS for operational or commercial use. 


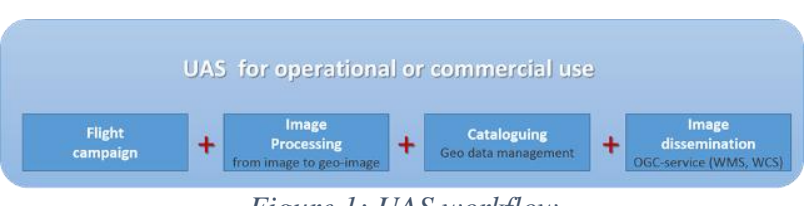

Figure 1: UAS workflow

\section{THE SELSAS-HYM PROJECT}

\subsection{Project scope and project partners}

Within this R\&D project a quasi-operational system for near real-time server-based aerial image processing and interpretation for high-resolution sensors was designed and realized. Focus is to deliver fast situation awareness information (near RT) to the soldier or remote experts. The innovations of this projects are

- a generic multi-sensor platform in the wing pod of a glider (UAV), with focus on hyper spectral imageries

- near RT image data preprocessing,

- near RT cataloguing and dissemination in ERDAS APOLLO

- distribution as OGC services and implementation of WPS for remote access

It was carried out by the cooperation of two industry partners and a defense research partner each with specific know-how. OHB System $\boldsymbol{A G}$ is a mid-size German space technology company, highly specialized for construction of satellites and aerial platforms.

$\boldsymbol{I O S B}$ stands for „Institute of Optronics, System Technologies and Image Exploitation" of the German Fraunhofer Institute, specialized in multi-sensory systems.

GEOSYSTEMS is the Intergraph Distribution Partner for the Geospatial Portfolio and solution provider for Geo-IT solutions in Germany.

\subsection{Hard- and Software components}

- Flight platform: CONDOR is an operational flight platform which facilitates real-time wide-range reconnaissance tasks at altitudes of up to $9 \mathrm{~km}$ on missions lasting 10 hours. It can operate manned as well unmanned. It is extreme low observable.

$$
\begin{aligned}
& \text { - Cameras: } \\
& \text { - Specim AISA EAGLE II } \\
& \text { - SVCam-SVS } 16000
\end{aligned}
$$

- Data link system (ARDS) from OHB with its efficient data management prepares sensor data for being transmitted by its data link with data rates of $83 \mathrm{Mbit} / \mathrm{s}$ (COFDM) or 247 $\mathrm{Mbits} / \mathrm{s}$ (single carrier) to the ground station over distances of up to $250 \mathrm{~km}$.

- Mobile ground control station for mission planning and control, sensor data exploitation, with interfaces to command and control environment

- Image processing and image dissemination: ERDAS IMAGINE and ERDAS APOLLO

- Automation of process workflow: GEOSYSTEMS METAmorph!IT

The flight platform, the data link system and the mobile ground control station is in the responsibility of $\mathrm{OHB}$, the wing-pod equipment and hyper spectral processing was the part of IOSB and the data preprocessing, data dissemination and process automation were realized by GEOSYSTEMS.

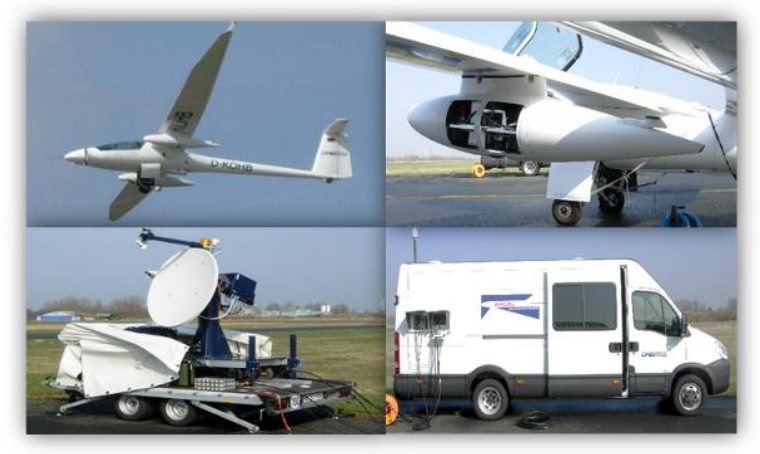

Figure 2: SELSAS-HyM Components -

UL: Glider Condor, UR: Wing pod with recording unit, $L L$ : data receiving antenna,

LR: mobile ground control station

\subsection{SELSAS-HyM system architecture}

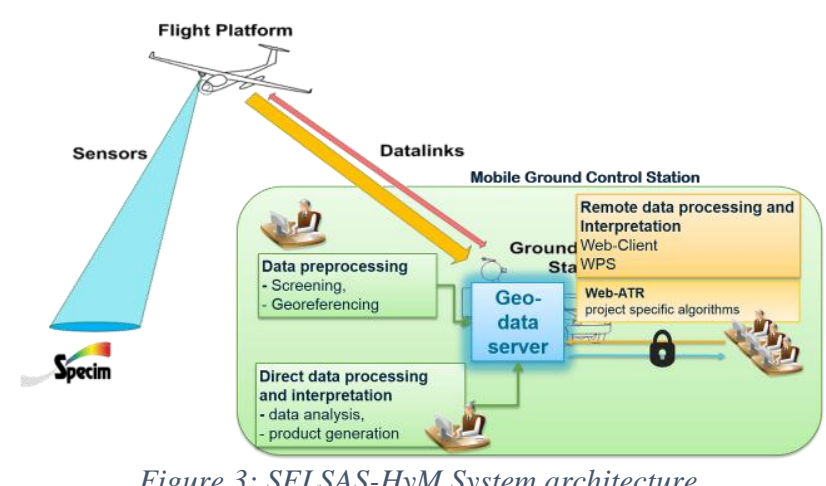

Figure 3: SELSAS-HyM System architecture

The carrier operates according the flight management tool. Via the data downlink unit (ARDS) the data are permanently downlinked to the mobile ground control station (via antenna). The incoming data will automatically be georeferenced according to the recorded IMU positions per image and mosaicked in 10 second units for easier handling. Other image processing algorithms would also be possible, if required. Per image an ISO 19115 metadata file is generated. Subsequently the data are catalogued in the geo data management system ERDAS APOLLO. At this step the metadata are stored in a database, the image data are decoded to be available as webservices for visualization (WMS or ECWP) or for download (WCS).

The automation of the data handling workflow provides the data from the downlink to the dissemination as OGC-web service in near real-time, with a rate of $1 \mathrm{sec} /$ image. This workflow is generic and independent from the SELSAS-Hym project. 


\section{AUTOMATED PROCESS FOR DATA HANDLING}

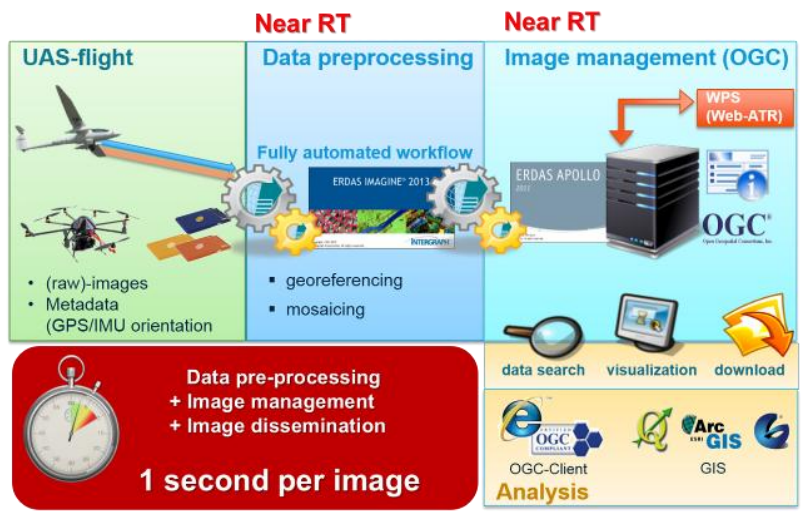

Figure 4: Automated workflow for data pre-processing to data dissemination

The incoming data, either via direct data downlink (SELSASHym) or after finishing the flight campaign (memory stick/card) will be read from the device. The corresponding metadata are converted into ISO 19115 standard. Data pre-processing function are applied to the imageries. The georeferencing is based on the delivered IMU orientation, which is re-calculated per image from yaw, pitch and roll into position $(\mathrm{x}, \mathrm{y}, \mathrm{z})$ and angle $(\Omega, \varphi, \kappa)$. The correction function is applied to the four corners of the image under consideration of a DEM at those corner points. This methodology achieves a reasonable results and has been applied due to the fact, that the near real-time approach for the SELSAS-HyM project was the driver. It should be noted, that photogrammetric algorithms could also be applied, but at the expense of the near real-time processing.

Functions of ERDAS IMAGINE are seamlessly batched and controlled by the workflow manager. The workflow manager catalogues the data into the geo data management system ERDAS APOLLO. Due to the crawling mechanism metadata will be ingested without any time lag into the catalogue. Instantly the data are made available as OGC services. Due to the streamlining of all processing with GEOSYSTEMS METAmorph!IT, a highly flexible and adaptable framework to perform workflow management tasks, a throughput of 1 sec/image is achieved.

The workflow was tested with online-downlink data of SELSAS-Hym as well with data delivered from an octocopter.

As soon the data are available as OGC-web services, it can be used for visualization or for analysis in GIS or image processing systems.

\section{WEB PROCESSING SERVICE (WPS)}

By implementing server-based geoprocessing, so-called web processing services (WPS), specific functions can be started from a web client to dynamically run those functions at a server. The result will be automatically catalogued and will be provided as web-service to the user.

This concept is available in the SELSAS-HyM project, with focus to image processing algorithms, supporting the ATR procedures. The advantage is, that a remote experts may contribute to a situation awareness picture by adding their knowledge to the stack of UAV-images, e.g. a change detection analysis. There are neither local images nor specific software necessary at the specialists' desktop - just a web client with WPS-functionality. A decision maker at any place will be provided with more image products for his decision.

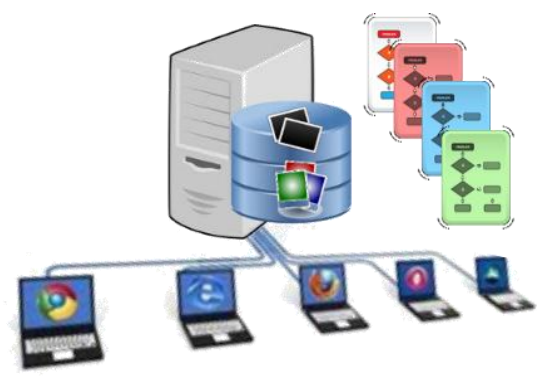

Figure 5: WPS concept

Figure 5 explains the WPS concept. Pre-processed image data and its metadata are stored in a geodata management. Specific algorithms are available as WPS. This is dynamically - new algorithms can be added or existing algorithms might be modified or removed. From various web clients with WPS capability any of the algorithms can be started, the necessary parameters can be adjusted. The algorithms runs on the server, the result will be catalogued and made available as web service through the catalogue to all users with permission to the system. The implementation of WPS provides clear advantages:

- One-time costs for Hard- and Software

- Flexible and extensible functions

- Centralized data storage

- Simple administration

\section{RESULTS}

\subsection{SELSAS-HyM example}

A typical example of a Condor-flight generates about 1600 VISimages, 11 bit with $4000 * 3000$ pixels per image.

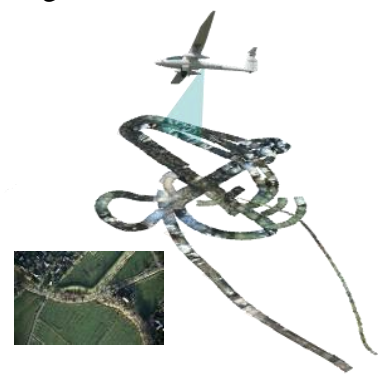

Figure 6: SELSAS-HyM example

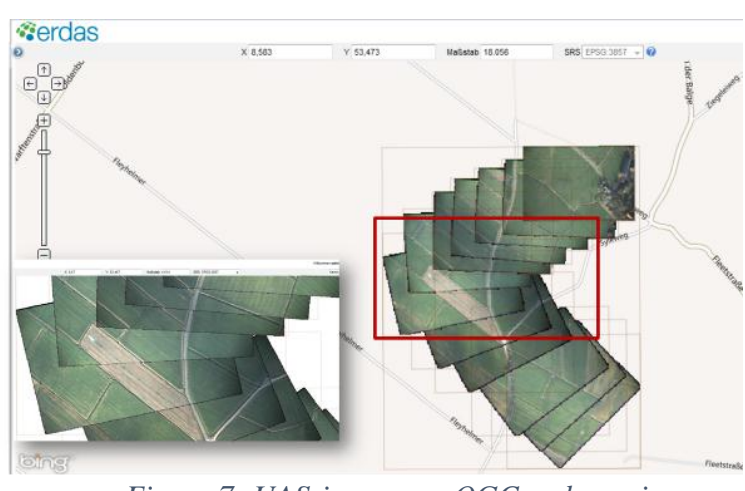

Figure 7: UAS-images as OGC-web service

Within this example the images have been pro-processed and then provided as OGC-web services. Figure 7 shows the georeferenced data superimposed to Bing maps. The 
enlargement states the geometric situation between the single georeferenced images, obeying the $1 \mathrm{sec} / \mathrm{image}$ algorithm, for the producing fast situation awareness information.

\subsection{Small UAV - octocopter}

The methodology was also applied to imageries of a small UAV, here an octocopter.

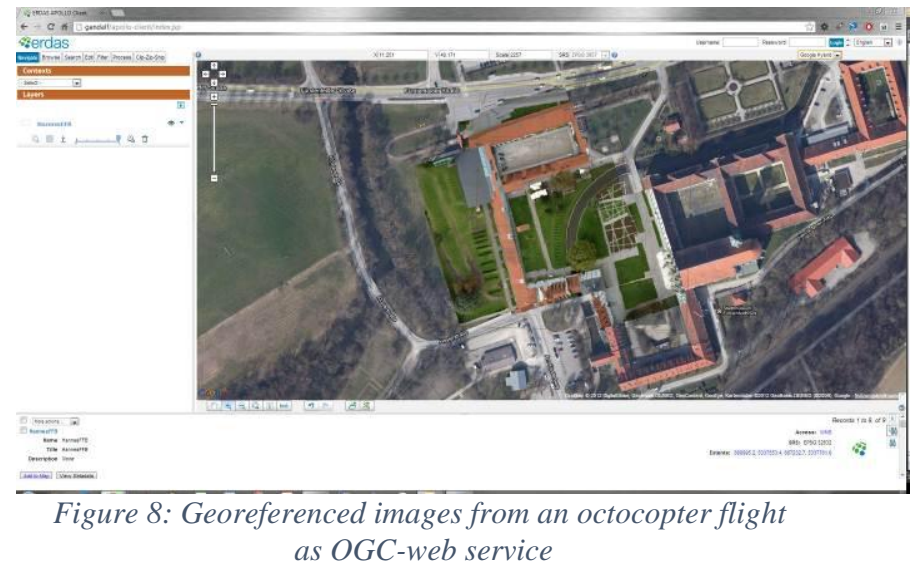

\section{CONCLUSION}

The SELSAS-Hym project was used to develop of a quasioperational real-time data awareness situation system based on existing components of a consortium with industrial and research partners. Beside project specific results, the automated and seamless workflow for data pre-processing and data management is a generic component, which completes the UAS for commercial and operational business models.

Example are surveillance of corridors, mass rallies, catastrophes, storm damages or as customer service for flight shops, as a rapid pre-checking of the image quality (e.g. flight campaign in remote places).

\section{ACKNOWLEDGEMENT}

Special thanks goes to the SELSAS-HyM team from OHB and IOSB and my colleagues Fritz Spitzer and Thomas Weser from GEOSYSTEMS GmbH. 